\title{
Frontières
}

\section{L’influence des circonstances du décès sur l'ajustement au deuil}

\author{
Hélène Thériault, Monique Séguin et Marc-Simon Drouin
}

Volume 24, numéro 1-2, automne 2011, printemps 2012

URI : https://id.erudit.org/iderudit/1013084ar

DOI : https://doi.org/10.7202/1013084ar

Aller au sommaire du numéro

\section{Éditeur(s)}

Université du Québec à Montréal

ISSN

1916-0976 (numérique)

Découvrir la revue

Citer cet article

Thériault, H., Séguin, M. \& Drouin, M.-S. (2011). L’influence des circonstances du décès sur l'ajustement au deuil. Frontières, 24(1-2), 45-54.

https://doi.org/10.7202/1013084ar
Résumé de l'article

La littérature scientifique explique que plusieurs variables peuvent influencer l'ajustement au deuil. La nature du décès - plus particulièrement un décès après suicide - $\mathrm{a}$ été identifiée comme une des variables pouvant contribuer à rendre le deuil plus complexe. La présente étude a pour but d'évaluer les réactions survenant plusieurs années après un décès par suicide $(n=31)$ et par maladie $(n=38)$ ainsi que le lien avec les facteurs contribuant au développement de complications. Les mesures incluaient des questionnaires auto-administrés évaluant les réactions des personnes endeuillées (échelle de dépression, traumatique, de détresse) ainsi que le style d'attachement. Seul le style d'attachement insécure et l'intensité des réactions traumatiques prédisent le développement d'un deuil compliqué, éléments sur lesquels il serait important d'intervenir. 


\section{Résumé}

La littérature scientifique explique que plusieurs variables peuvent influencer l'ajustement au deuil. La nature du décès - plus particulièrement un décès après suicide a été identifiée comme une des variables pouvant contribuer à rendre le deuil plus complexe. La présente étude a pour but d'évaluer les réactions survenant plusieurs années après un décès par suicide $(n=31)$ et par maladie $(n=38)$ ainsi que le lien avec les facteurs contribuant au développement de complications. Les mesures incluaient des questionnaires auto-administrés évaluant les réactions des personnes endeuillées (échelle de dépression, traumatique, de détresse) ainsi que le style d'attachement. Seul le style d'attachement insécure et l'intensité des réactions traumatiques prédisent le développement d'un deuil compliqué, éléments sur lesquels il serait important d'intervenir.

Mots clés: deuil - suicide - complications attachement.

\section{Abstract}

In the literature, there have been many studies devoted to adjustment to bereavement as well as to the variables related to it. The objective of our study is to determine and compare mourners' reactions many years after their loss as well as the factors generating complications, using two samples: death by suicide $(n=31)$ and death due to sickness ( $n=38)$. Self-administered questionnaires were used in our inquiry to measure mourners' reactions to death (depression, traumas, and distress) and their bond style with the deceased person. We believe that two factors (variables) could lead to a complicated bereavement and necessitate an intervention: an insecure bond style and/or intense traumatic reactions.

Key words: grief - suicide complications - attachment.

\section{L'influence des circonstances du décès sur l'ajustement au deuil}

\author{
Hélène Thériault, M.Ps., \\ candidate au Psy. D., UQO et Groupe McGill d'études \\ sur le suicide.
}

Monique Séguin, Ph. D.,

UQO et Groupe McGill d'études sur le suicide.

Marc-Simon Drouin, UQAM.

\section{LES DÉCÈS AU QUÉBEC}

La mort fait partie intégrante du cycle de l'existence, faisant chaque année des milliers de personnes endeuillées par la perte d'un être cher. En effet, selon Statistique Canada, de 2008 à 2009, 242863 personnes ont perdu la vie au Canada, dont 56700 au Québec (Institut national de santé publique du Québec, 2005). Même si elle est commune, l'expérience du deuil s'avère généralement douloureuse et constitue un événement de vie des plus stressants (Reed, 1998). Alors que la plupart des personnes endeuillées connaîtront un processus de deuil normal, évoluant graduellement d'un choc initial accompagné d'une détresse vers une acceptation et une intégration de la perte, certaines personnes peuvent dévier de cette trajectoire prévisible et vivre un deuil se distinguant par son intensité et par sa persistance dans le temps (Enright et al., 2002). En effet, plusieurs auteurs (Bonanno et al., 2004; Prigerson et al., 1995) estiment que de $10 \%$ à $20 \%$ des personnes endeuillées manifestent des réactions intenses liées au deuil, lesquelles se prolongent, occasionnant des difficultés à s'ajuster à la perte. Dans la littérature sur le deuil, les principales difficultés répertoriées en termes de complications liées au deuil sont les suivantes: des réactions et préoccupations intenses et prolongées liées au deuil, de la détresse psychologique, des réactions dépressives et anxieuses notables Harwood et al., 2002 ; Kitson, 2000; Grad et Zavasnik, 1999; Cleiren et al., 1996; Cleiren et al., 1994; Séguin et al. 1995a, 1995b; Farberow et al., 1992; Range et Niss, 1990).

Afin de tenter de repérer les individus endeuillés manifestant de multiples 
complications lors de leur processus de deuil, des chercheurs se sont intéressés à différentes variables: celles liées aux conditions précédant le décès (la nature de la perte selon le lien avec le défunt, les antécédents de santé mentale), celles liées aux circonstances du deuil (l'âge de la personne endeuillée, celui de la personne décédée, l'impact d'une mort soudaine versus une mort anticipée, la mort par suicide, par accident, par homicide, etc.) et, enfin, celles survenant à la suite du décès (le soutien social, les événements de vie, la présence de conflits au sein de la famille ou de l'entourage, etc.). Certaines de ces variables peuvent influencer l'ajustement à un deuil et occasionner des complications. Parmi les circonstances entourant le décès, la mort à la suite d'un suicide a souvent été identifiée comme un des facteurs de risques pouvant contribuer à un deuil compliqué.

\section{ÉPIDÉMIOLOGIE DU DEUIL À LA SUITE D'UN SUICIDE}

Le suicide a donc fait l'objet d'une multitude de recherches et d'études dans le but d'identifier, de comprendre, de prédire et surtout de prévenir ce phénomène. Au cours des dernières décennies, l'attention s'est également portée sur les personnes endeuillées par suicide (Barrett et al., 1990). Le suicide est un problème important de santé publique. D'après les données disponibles, en 2008 , on rapportait 1103 décès par suicide au Québec. Cette province affiche d'ailleurs le plus haut taux de décès par suicide de toutes les provinces canadiennes, et ce, tant chez les hommes que chez les femmes. D'après les études, on estime que, pour chaque suicide, il y a environ de 6 à 28 personnes qui sont touchées, vivant ainsi le deuil d'un proche (Knieper, 1999; Shneidman, 1969). À la lumière de ces données, il y aurait, au moins, plus de 6000 endeuillés par suicide au Québec au cours d'une seule année.

Dans la littérature sur le deuil, les premières études, pour la plupart fondées sur des observations cliniques et des études de cas (Osterweiss et al., 1984; Rudestam, 1977 ; Wallace, 1973, cité par Rudestam, 1977; Cain et Fast, 1972, cité par Calhoun, Selby et Selby, 1982; Shepherd et Barraclough, 1974; Sheidman, 1969) effectuées avec des personnes endeuillées par suicide, ont expliqué l'unicité de ce décès par la complexité ou l'intensité de certaines réactions et ont également souligné que ce type de deuil serait le plus difficile à vivre. Ces opinions suggéraient que le suicide d'un proche entraînerait des difficultés considérables dans le processus d'ajustement au deuil, et la plupart des auteurs rapportaient plus de réactions symptomatologiques chez les endeuillés par suicide.

Sur la base d'études plus rigoureuses que celles mentionnées précédemment (Grad et Zavasnik, 1999; Séguin et al. 1995a, 1995b ; Cleiren, 1993 ; McIntosh et Kelly, 1992 ; Barrett et Scott, 1990; Farberow et al., 1987 ; Demi, 1984), des chercheurs ont comparé des personnes endeuillées par suicide à des groupes de contrôle, tout en ajoutant plusieurs temps de mesures, des outils plus spécifiques, etc., d'où des conclusions plus probantes ont émergé. Parmi celles-ci, certaines études (Séguin et al., 1995; Farberow et al., 1987) constatent une diminution considérable de la symptomatologie avec le passage du temps, si bien que les différences observées entre les groupes d'endeuillés à la suite d'un suicide sont quasi imperceptibles après une certaine période de temps (McIntosh 1993; Barrett et al., 1990).

De plus, plusieurs études comparatives (de Groot et al., 2006; Dyregrov et al., 2003; Murphy et al., 2003; Harwood et al., 2002; Kitson, 2000; Bailley et al., 1999; Grad et Zavasnik, 1999; Reed, 1998; Cleiren et al., 1996; Séguin et al., 1995a, 1995b; Cleiren et al., 1994 ; Farberow et al., 1992; Silverman et al., 1994-1995 ; Barrett et al., 1990; McNiel et al., 1988) observent les mêmes résultats et constatent qu'il y a plus de ressemblances que de différences entre les groupes d'endeuillés (morts par suicide, par accident, morts naturelles soudaines ou anticipées) au niveau de la symptomatologie et de ses manifestations (dépression, anxiété, détresse subjective, signes de deuil compliqué et ajustement à la perte), particulièrement lorsqu'il s'agit de décès traumatiques (accident, homicide, etc.).

Le deuil par suicide semble demeurer une expérience qualitativement complexe (Ellenbogen et Gratton, 2001). En plus des préoccupations et des réactions émotionnelles intenses liées au deuil après suicide, certaines recherches (Kaltman et Bonanno, 2003) ont mis l'accent sur des complications particulières lors de décès violents. La présence simultanée d'un deuil et d'un traumatisme pourrait entraîner des difficultés d'ajustement plus grandes et plus prolongées (Prigerson et al., 1999), à cause de la présence d'une dépression majeure, accompagnée d'un trouble de stress traumatique. "Apprendre la mort soudaine ou violente d'un être cher» constitue un "stresseur traumatique extrême»(APA, 1994, traduction libre: 424) qui s'accompagne généralement d'une détresse importante. Pourtant, il est surprenant, comme le soulignent Kaltman et Bonanno (2003), que les symptômes post-traumatiques aient été, jusqu'à récemment, peu évalués dans les études sur le deuil par suicide (Séguin et al., 1995 ; Reed, 1993 ; Barrett et al., 1990). La pertinence d'évaluer cette variable semble pourtant dénoncée par plusieurs auteurs (Kaltman et al., 2003; Callahan 2000; Bonanno et al., 1999; Cleiren et al., 1995). Même si les endeuillés par suicide n'ont pas été témoins du décès violent, ils peuvent imaginer les scénarios entourant le décès et peuvent être hantés par des images intrusives et troublantes (Séguin et Fréchette, 1999), d'où la pertinence d'évaluer ce registre de symptômes.

La présence de ces complications potentielles chez certains endeuillés, indépendamment de la nature du décès, nous amène à nous questionner sur les raisons pour lesquelles un individu en développera, alors qu'un autre s'adaptera sans complication à cette perte. Comme le démontrent les études comparatives effectuées auprès des endeuillés par suicide et mentionnées précédemment, la nature du décès ne semble pas constituer une variable qui puisse expliquer la présence ou l'absence de complications lors du processus de deuil. Si ce n'est donc pas la nature du décès qui peut déterminer la présence de complications chez certains endeuillés, quelle autre variable pourrait s'avérer plus importante? Les endeuillés par suicide ne constituent pas un groupe homogène, et il pourrait exister chez eux certains sous-groupes montrant davantage de vulnérabilité, une fragilité présente avant même le deuil (Séguin et al., 2006; Séguin et al., 1994). Parmi ces vulnérabilités, certaines recherches ont principalement identifié la présence d'un style d'attachement insécure (Vanderwerker et al., 2006; Stroebe et al. 2005a, 2005b; Mulkulincer et al., 1993) et la présence d'antécédents de troubles de santé mentale chez certains endeuillés (Brent et al., 2008; Stenager et al., 2008; Tsuchiya et al., 2005; Jordan, 2001; Egeland et al., 1990) pouvant les prédisposer au développement de complications à la suite de leur deuil (Silverman et al., 2001; Séguin et al., 1995; Séguin et al., 1994).

Des études (Mitchell et al., 2004; Cleiren et al., 1994; Cleiren, 1993 ; Reed et Greenwald, 1991) ont démontré que la nature de la relation se révélait être une variable qui contribuait de manière significative à la prédiction de l'issue du deuil. On a d'ailleurs suggéré que la relation d'attachement préexistante et le style d'attachement de l'endeuillé pourraient déterminer la façon dont la personne transigerait avec le détachement et comment elle s'ajusterait à cette perte (Mitchell et al., 2004 ; Cleiren et al., 1996; 
Séguin et al., 1995a, 1995b ; Cleiren et al., 1994; Cleiren, 1993; McIntosh et Kelly, 1992; Reed et Greenwald, 1991; Barrett et Scott, 1990). Toutefois, cette variable le style d'attachement - reste encore une variable négligée, donc peu évaluée dans les recherches. De plus, certaines études réalisées sur le deuil suggèrent que les personnes présentant un style d'attachement insécure (évitant, préoccupé ou craintif) étaient plus susceptibles d'avoir des difficultés d'ajustement aux expériences de perte (Vanderwerker et al., 2006; Stroebe et al. 2005a, 2005b; Mulkulincer et al., 1993), de manifester des réactions dépressives et/ou anxieuses plus importantes et plus de réactions somatiques (Wayment et Vierthaler, 2002; Field et Sundin, 2001; Mulkulincer et al., 1993). Les individus ayant un attachement sécure manifestent, quant à eux, des réactions liées au deuil moins intenses (Van Doorn et al., 1998), moins de réactions anxieuses, dépressives et traumatiques entre 4 et 18 mois après le décès de leur époux (Fraley et Bonanno, 2004; Wayment et Vierthaler, 2002) et ils sont aussi plus enclins à rechercher du soutien lors d'événements stressants ou lors de pertes (Simpson et al., 1992).

À la suite de ces études, il nous semble important, tant sur le plan de la recherche qu'au point de vue clinique, de mieux étudier les liens qui existent entre la présence de ces sous-groupes, le développement de complications liées au deuil et le style d'attachement.

\section{LES QUESTIONS DE RECHERCHE}

Dans un premier temps, la présente étude vise à explorer, chez les endeuillés, si la nature du décès, par suicide ou par maladie, influence certaines réactions liées au deuil, à la détresse psychologique, à la présence de réactions dépressives et post-traumatiques répertoriées dans la littérature, et ce, plusieurs années après le décès. Cette étude souhaite également souligner la présence de certaines préoccupations liées au deuil dans chacun des groupes.

Dans un deuxième temps, cette étude souhaite vérifier l'hypothèse selon laquelle il existerait des sous-groupes éprouvant des réactions de deuil complexes et ainsi explorer les différences entre ces groupes par le biais des variables nommées précédemment.

Enfin, cette étude veut mettre en lumière les variables qui sont significativement associées à la présence de réactions complexes liées au deuil parmi celles présentes avant le décès (style d'attachement) et celles survenant après la mort (détresse psychologique, réactions dépressives, réactions post-traumatiques).

\section{MÉTHODOLOGIE}

\section{LES PARTICIPANTS}

Soixante-neuf personnes adultes endeuillées par la perte d'un proche ont été recrutées entre avril 2008 et février 2009 au Québec. Il s'agissait de 31 personnes endeuillées à la suite d'un décès par suicide ( 7 hommes et 24 femmes) et de 38 personnes endeuillées par un décès consécutif à une maladie (14 hommes et 24 femmes). La perte avait eu lieu de 2 à 7 ans avant l'étude, et ces personnes étaient toutes des proches du défunt (conjoint[e], fratrie ou membres de la famille immédiate). Près d'un cinquième des participants de cette étude avait été affecté par le décès d'une même personne. Étant donné que les réactions au deuil sont individuelles, ces participants ont été inclus dans l'étude.

\section{LES PROCÉDURES}

Les individus endeuillés à la suite d'un décès par suicide ont été recrutés dans le Groupe McGill d'études sur le suicide. Ces participants ont été sélectionnés de façon aléatoire à l'aide d'archives répertoriant des personnes ayant déjà participé à des recherches réalisées par des chercheurs du Groupe McGill d'études sur le suicide. Lors d'études antérieures, les personnes avaient accepté d'être contactées à nouveau pour participer à d'autres projets de recherche, comme indiqué dans le formulaire de consentement initial. Toutes les personnes ayant participé à cette nouvelle étude ont signé un formulaire de consentement.

Les participants endeuillés à la suite d'un décès par mort naturelle ont, quant à eux, été recrutés par l'entremise des principaux organismes offrant des services d'aide et de soutien aux personnes endeuillées (Lumi-Vie, Deuil-Secours, etc.). Ces organismes ont accepté de joindre à leur envoi postal régulier destiné à leurs membres, une lettre expliquant le projet de recherche. De plus, étant donné le nombre très limité de participants recrutés par l'intermédiaire de ces organismes $(n=6)$, nous avons aussi utilisé la technique d'échantillonnage «boule de neige» pour recruter d'autres personnes endeuillées.

Les personnes désireuses de participer à ce projet de recherche devaient communiquer avec l'équipe de recherche. La personne responsable de la recherche expliquait les paramètres de l'étude et sollicitait un rendez-vous avec les participants. Généralement, les rendez-vous étaient fixés au domicile des participants. À la suite de la signature du formulaire de consentement, les participants remplissaient une série de questionnaires d'une durée d'environ 90 minutes, portant sur leurs caractéristiques sociodémogra- phiques ainsi que leurs réactions au deuil. Certains questionnaires ont parfois été remplis lors d'un entretien téléphonique $(n=6)$ ou envoyés par la poste $(n=26)$, étant donné les disponibilités limitées de certains participants ainsi que l'éloignement de leur lieu de résidence. Ce projet de recherche a reçu l'aval du Comité d'éthique de la recherche de l'Université du Québec en Outaouais.

\section{LES MESURES}

\section{LES VARIABLES \\ SOCIODÉMOGRAPHIQUES}

Ce questionnaire était composé de 14 questions visant à décrire les caractéristiques sociodémographiques des participant(e)s (l'âge, le sexe, la nationalité, la profession du participant, etc.). Il contenait également des questions sur la personne décédée (par exemple, son âge, son lien de parenté avec le ou la participant[e], le type de décès et le nombre de mois écoulés depuis sa mort).

\section{LES VARIABLES PSYCHOLOGIQUES}

Questionnaire sur l'expérience du deuil (Grief Experience Questionnaire) (GEQ)

(Barrett et Scott, 1989; Vallerand, 1989)

Le Grief Experience Questionnaire évalue 11 dimensions du deuil, dont les réactions somatiques, les réactions de deuil, la recherche d'explication, la perte de soutien social, la stigmatisation, la culpabilité, la responsabilité, la honte, le rejet, les comportements autodestructeurs et les réactions uniques (Barrett et Scott, 1989). Il contient 55 énoncés pour lesquels les participants doivent identifier sur une échelle de 1 (pas du tout) à 5 (toujours) l'intensité de leurs réactions. Il a une excellente fidélité générale $(\alpha=0,97)$, variant de 0,68 à 0,89 pour chacune des dimensions. Sa traduction française jouit également d'une excellente validité interne ( $\alpha=0,96$; Vallerand, 1989). Ce questionnaire a été choisi, car il est l'un des principaux instruments de mesure pour le deuil (Barrett et Scott, 1989); il intègre également des éléments spécifiques du deuil à la suite d'un décès par suicide et nous permettra de comparer nos résultats à ceux des études antérieures.

\section{Inventaire de dépression de Beck}

Ce questionnaire évalue la présence et l'intensité de la dépression dans une dimension cognitive, affective et somatique (Bernier et Pietrulewicz, 1997). Il est composé de 21 items dont les énoncés décrivent la gravité du symptôme dépressif sur une échelle de 5 points. Les participants doivent choisir l'énoncé traduisant 
le mieux leur vécu au cours de la semaine. Un score total de 0 à 9 indique une absence de dépression; de 10 à 18, une dépression légère à modérée; de 19 à 29, une dépression modérée à grave, et de 30 et plus, une dépression très intense (Bernier et Pietrulewicz, 1997). Ce questionnaire a une bonne fiabilité (Gauthier et al., 1982) et une fidélité test-retest (Bernier et Pietrulewicz, 1997). La version canadienne-française a également une très bonne cohérence interne pour les échantillons cliniques et non cliniques $(0,93$ et 0,92).

\section{Échelle révisée de l'impact}

des événements (Impact of Event

Scale-Revised)

Ce questionnaire est composé de 22 items, répartis en trois sous-échelles mesurant les symptômes d'évitement, d'intrusion et d'activation neurovégétative à la suite d'événements traumatiques. La traduction française de ce questionnaire a de bonnes qualités psychométriques (Brunet et al., 2003). En effet, la cohérence interne $(0,93$ pour le score global et 0,81 à 0,86 pour les sous-échelles) ainsi que la stabilité test-retest après trois mois sont excellentes pour le score global $(r=0,76)$ et de $r=0,71$ à 0,77 pour les sous-échelles (Cuillerier, 2004).

\section{Questionnaire de l'indice de détresse}

psychologique (IDPESQ-14) (Préville,

Boyer, Potvin, Perrault, et Legaré, 1992)

Ce questionnaire évalue la détresse psychologique actuelle des participants de l'étude et reflète ainsi la santé psychologique de la personne. Il a été adapté du Psychiatric Symptoms Index d'IIfeld (1976). Il comprend 14 énoncés, répartis en 4 dimensions, mesurant la fréquence des symptômes liés à la dépression, à l'anxiété, aux troubles cognitifs et à l'irritabilité. Les participants doivent évaluer l'intensité des différents symptômes sur une échelle de 0 à 3, allant de jamais (0) à très souvent (3). Un score élevé indique un niveau de détresse élevé. Le questionnaire montre une excellente consistance interne ( $\alpha=0,89$; Préville et al., 1992) et une validité de structure satisfaisante. Il a été traduit, validé en français ainsi qu'adapté et utilisé dans le cadre des Enquêtes Santé Québec 1987, 1992 et 1998, et dans plusieurs autres études auprès de la population québécoise.

Questionnaire sur les styles relationnels

(The Relationship Questionnaire)

Ce questionnaire, créé par Bartholomew et Horowitz (1991), utilise la typologie issue du modèle d'attachement de Hazan et
Shaver (1987). Quatre styles d'attachement (sécure "secure»; évitant «dismissing»; préoccupé «preoccupied» [nommé aussi "ambivalent» selon d'autres typologies]; craintif «fearful» [nommé aussi «désorganisé » d'après d'autres typologies]) sont décrits en fonction des caractéristiques relationnelles et de l'évaluation positive ou négative de soi et des autres. Dans la première partie de ce questionnaire, le participant doit choisir, parmi les quatre styles d'attachement, la description du style d'attachement auquel il s'identifie le plus. Dans la deuxième partie, il doit indiquer, sur une échelle de 1 à 7 (allant de fortement en désaccord à fortement en accord), jusqu'à quel point la description de chacun des quatre styles d'attachement correspond à la perception qu'il a de lui-même. Cet outil a été utilisé dans plusieurs recherches québécoises (De Montigny, 2002), en raison de ses qualités psychométriques. En effet, il a démontré des coefficients de consistance interne variant de 0,49 à 0,71 pour les quatre styles d'attachement (Scharfe et Bartholomew, 1994). Il a également présenté une stabilité temporelle moyenne lors d'un suivi de huit mois auprès d'une population étudiante (Scharfe et Bartholomew, 1994). La validité de structure a été confirmée par la comparaison avec d'autres modes d'évaluation (par exemple, autoévaluation vs évaluation par un ami, un partenaire amoureux ou des juges spécialisés; Griffin et Bartholomew, 1994). Enfin, la validité discriminante de l'instrument indique que les quatre styles d'attachement sont significativement différents et indépendants (Bartholomew et Horowitz, 1991).

\section{LES ANALYSES DES DONNÉES}

Des analyses descriptives ont d'abord été effectuées sur les différentes variables démographiques ainsi que sur les circonstances du deuil.

Le style d'attachement comprenait, au départ, quatre catégories (sécure, évitant, préoccupé et craintif), qui ont été réunies de manière à former deux catégories distinctes, soit un «style d'attachement insécure» et un "style d'attachement sécure » pour les analyses finales. En ce qui concerne les réactions post-traumatiques, étant donné qu'il n'existe pas de seuil permettant de distinguer les participants ayant des réactions plus intenses, elles ont été déterminées en fonction des valeurs supérieures à un écart type se situant au-dessus de la moyenne (score total > 31).

Dans un premier temps, des $\mathrm{khi}^{2}$ ont été effectués afin d'évaluer si les réactions liées au deuil, les réactions dépressives, les réactions post-traumatiques et de détresse psychologique ainsi que le type d'attachement différaient selon la nature du décès (suicide vs maladie).

Dans un deuxième temps, étant donné que seule la variable des réactions liées au deuil était statistiquement significative, nous avons repris cette variable en fonction du résultat du questionnaire Grief Experience Questionnaire pour former deux groupes distincts selon les réactions des endeuillés («réactions saines» vs «réactions complexes»), et ce, pour chacun des deux groupes («suicide» vs «maladie»). Il est important de noter que ce questionnaire fut conçu spécifiquement pour les personnes endeuillées par suicide et qu'il s'avère donc plus sensible à détecter des différences chez ce groupe d'endeuillés. L'auteur de ce questionnaire n'ayant pas établi de seuil entre "des réactions saines» et "des réactions complexes", nous avons choisi de l'établir à un écart type se situant au-dessus de la moyenne. Les participants ayant obtenu des scores se situant à un écart type au-dessus de la moyenne (score total $>124,9$ ) dans ce questionnaire ont été identifiés comme présentant des réactions complexes $(n=14)$, alors que les autres participants ont été considérés comme expérimentant des réactions saines $(n=55)$. Afin d'évaluer les différences en fonction de l'ajustement du deuil, des anova simples ont été répétés, en se basant sur le score moyen retrouvé pour les mêmes caractéristiques psychologiques. Le seuil de signification a été fixé à $\mathrm{p}<0,05$ pour toutes les analyses. Pour mesurer le style d'attachement, des $\mathrm{Khi}^{2}$ ont été effectués étant donné qu'il s'agissait d'une variable catégorielle.

Nous avons aussi regroupé les participants ayant un deuil sain $(n=55)$ et ceux ayant un deuil complexe $(n=14)$, indépendamment de la nature du décès, pour mesurer (par le biais d'anova) entre ces deux groupes les différences concernant les variables suivantes: réactions dépressives, post-traumatiques et détresse psychologique.

Enfin, une régression logistique pas à pas a été menée afin d'évaluer si la symptomatologie (réactions liées au deuil, réactions post-traumatiques, réactions de détresse psychologique, nature du deuil ainsi qu'attachement) prédisait le développement de complications dans le processus du deuil. Le seuil de signification a été fixé à $\mathrm{p}<0,05$ pour toutes les analyses.

\section{LES RÉSULTATS}

\section{LE PROFIL SOCIODÉMOGRAPHIQUE} DES PARTICIPANTS

Les participants étaient, en moyenne, âgés de 48 ans $(\mathrm{ET}=12,25)$ chez les personnes endeuillées après un décès par 
suicide (ES : endeuillés après un décès par suicide) et de 53 ans $(E T=14,62)$ chez les personnes endeuillées après un décès par maladie (EA: endeuillés par mort anticipée). La grande majorité était des femmes $(77,4 \%$ pour ES et $63,2 \%$ pour EA). La plupart d'entre elles étaient mariées ou vivaient en union de fait, elles occupaient un emploi et avaient une scolarité de niveau collégial, professionnel ou supérieur. Aucune différence significative n'a été observée entre les deux groupes quant aux caractéristiques sociodémographiques.

Des différences significatives ont été observées quant à l'âge des personnes décédées, au nombre de mois depuis le décès et au lien de parenté. Les personnes EA étaient significativement plus âgées (ES: en moyenne 45,8 ans vs EA: en moyenne 70,8 ans), et le deuil était plus récent comparativement aux personnes ES (ES : 71,3 mois vs EA : 40,3 mois). Les participants ES étaient majoritairement des membres de la fratrie (ES : $54,8 \%$ vs EA: $15,8 \%$ ), alors que les participants EA étaient surtout des enfants de parents décédés (ES: 19,4\% vs EA: 63,2\%). Le nombre de conjoints ou de conjointes était comparable dans les deux groupes (ES: $25,8 \%$ vs EA : $21,2 \%$ ). Enfin, plus de $60 \%$ des EA connaissaient le diagnostic médical de leur proche depuis plus de 6 mois : cancer $(65,8 \%)$, maladie d'Alzheimer $(15,8 \%)$ ou maladie chronique $(10,5 \%)$.

La symptomatologie et la nature

du décès (suicide et maladie)

(tableaux 1 et 2)

Les $\mathrm{khi}^{2}$ ont révélé une association significative entre la nature du décès et les réactions liées au deuil $\left(\chi^{2}(1)=4,99\right.$; $\mathrm{p}<0,05)$. Il semblerait que les personnes endeuillées par suicide seraient plus nombreuses à avoir des préoccupations intenses (complexes) liées au deuil, à la suite du décès d'un proche $(32,3 \%$ vs $10,5 \%)$. Afin de mieux comprendre les différences observées entre les deux groupes (voir le tableau 1), nous avons examiné chacune des sous-échelles du questionnaire de deuil à l'aide d'analyses de variances simples. Après correction pour les comparaisons multiples (Bonferroni), seules les échelles suivantes distinguaient les deux groupes: la recherche d'explications $(\mathrm{F}(1,67)=15,71 ; \mathrm{p}>0,05)$; les sentiments de responsabilité $(\mathrm{F}(1,67)=8,56$; $\mathrm{p}>0,05)$; les sentiments de rejet $(\mathrm{F}(1,67)$ $=14,95 ; p>0,05)$. Les réactions uniques $(\mathrm{F}(1,67)=34,67 ; \mathrm{p}>0,05)$ étaient plus présentes chez les endeuillés après un suicide.

Par ailleurs, aucune différence significative n'a été observée entre les deux groupes
TABLEAU 1

SCORE DES SOUS-ÉCHELLES DU QUESTIONNAIRE SUR L'EXPÉRIENCE DU DEUIL, SELON LA NATURE DU DÉCÈS

\begin{tabular}{lrrrrr}
\hline N $=69$ & \multicolumn{2}{c}{$\begin{array}{c}\text { Endeuillés } \\
\text { par suicide } \\
n=31\end{array}$} & \multicolumn{2}{c}{$\begin{array}{c}\text { Endeuillés } \\
\text { par maladie } \\
n=38\end{array}$} \\
Mesures & $\mathrm{M}$ & $\mathrm{ET}$ & $\mathrm{M}$ & $\mathrm{ET}$ & $\mathrm{p}$ \\
Réactions somations & 10,1 & 3,6 & 9,1 & 3,4 & 0,23 \\
Recherche d'explication & 13,7 & 5,0 & 9,4 & 4,3 & $0,00^{*}$ \\
Perte de soutien social & 7,8 & 4,2 & 6,9 & 3,1 & 0,32 \\
Stigmatisation & 8,6 & 3,9 & 6,7 & 2,8 & 0,03 \\
Culpabilité & 12,6 & 5,6 & 9,4 & 4,5 & 0,01 \\
Responsabilité & 9,7 & 4,8 & 6,6 & 2,4 & $0,00 *$ \\
Honte & 8,6 & 4,0 & 6,4 & 2,2 & 0,01 \\
Rejet & 10,1 & 5,1 & 6,4 & 3,0 & $0,00^{*}$ \\
Comportements & 8,3 & 3,4 & 6,9 & 2,5 & 0,05 \\
autodestructeurs & 10,7 & 3,2 & 6,9 & 2,1 & $0,00^{*}$ \\
Réactions uniques & 9,7 & 3,5 & 7,3 & 3,1 & $0,00^{*}$ \\
Réactions générales deuil & & & & & \\
\hline
\end{tabular}

$* \mathrm{p}<0,005$

TABLEAU 2

SYMPTOMATOLOGIE ET STYLE D'ATTACHEMENT SELON LA NATURE DU DÉCÈS

\begin{tabular}{ccc}
\hline & Endeuillés & Endeuillés \\
par suicide & par maladie \\
$n=31$ & $n=38$
\end{tabular}

\section{Mesures}

Légère

Moyenne à sévère

M ET

Dépression

Aucune dépression

Légère à sévère

Réactions traumatiques

$<1$ ET

$>1 \mathrm{ET}$

11

ET

$\mathrm{M}$

M

ET

$\mathrm{p}$

0,436

Réactions de deuil

Saines $<1$ ET

Complexes $>1$ ET

$11 \quad 35,5$

17

44,7

20

64,5

21

55,3

$\begin{array}{rrrr}24 & 77,4 & 32 & 84,1 \\ 7 & 22,6 & 6 & 15,8\end{array}$

0,473

\section{Style d'attachement}

Sécure

Insécure

$* \mathrm{p}<0,05$ 
TABLEAU 3

SYMPTOMATOLOGIE, STYLE D'ATTACHEMENT, NATURE DU DÉCÈS (SUICIDE VS MALADIE) ET AJUSTEMENT AU DEUIL «RÉACTIONS SAINES » VS «RÉACTIONS COMPLEXES »

\begin{tabular}{|c|c|c|c|c|c|c|}
\hline \multirow[b]{3}{*}{ Mesures } & \multicolumn{2}{|c|}{$\begin{array}{c}\text { Endeuillés } \\
\text { par suicide } \\
n=31\end{array}$} & \multicolumn{4}{|c|}{$\begin{array}{c}\text { Endeuillés } \\
\text { par maladie } \\
n=38\end{array}$} \\
\hline & $\begin{array}{c}\text { Réactions } \\
\text { saines } \\
n=21\end{array}$ & $\begin{array}{l}\text { Réactions } \\
\text { complexes } \\
n=34\end{array}$ & & $\begin{array}{c}\text { Réactions } \\
\text { saines } \\
n=34\end{array}$ & $\begin{array}{c}\text { Réactions } \\
\text { complexes } \\
n=4\end{array}$ & \\
\hline & M & M & $\mathrm{p}$ & M & M & $\mathrm{p}$ \\
\hline Détresse psychologique & 22,1 & 27,1 & 0,104 & 9,1 & 3,4 & 0,104 \\
\hline Dépression & 3,4 & 12,4 & $0,009 *$ & 9,4 & 4,3 & $0,009 *$ \\
\hline $\begin{array}{l}\text { Réactions } \\
\text { post-traumatiques }\end{array}$ & 22,9 & 30,6 & $0,006^{*}$ & 6,9 & 3,1 & $0,006^{*}$ \\
\hline
\end{tabular}

\begin{tabular}{lrrrrrrrrrl}
\hline Style attachement & & & & & & & & & & \\
Sécure & 19 & 61,3 & 4 & 12,9 & $0,003^{*}$ & 25 & 65,8 & 2 & 5,3 & $0,000^{*}$ \\
Insécure & 2 & 6,5 & 6 & 19,4 & 0,289 & 9 & 23,7 & 2 & 5,3 & 0,065 \\
\hline
\end{tabular}

$* \mathrm{p}<0,05$

\section{TABLEAU 4}

SYMPTOMATOLOGIE ET AJUSTEMENT AU DEUIL

\begin{tabular}{lccc}
\hline & $\begin{array}{c}\text { Endeuillés } \\
\text { par suicide } \\
n=31\end{array}$ & $\begin{array}{c}\text { Endeuillés } \\
\text { par maladie } \\
n=38\end{array}$ & \\
Mesures & $\mathrm{M}$ & $\mathrm{M}$ & $\mathrm{p}$ \\
$\begin{array}{l}\text { Détresse psychologique } \\
\text { Dépression }\end{array}$ & 22,3 & 27,2 & $0,017^{*}$ \\
$\begin{array}{l}\text { Réactions } \\
\text { post-traumatiques }\end{array}$ & 4,8 & 12,2 & $0,001^{*}$ \\
\hline
\end{tabular}

$* \mathrm{p}<0,05$

quant aux réactions dépressives, aux réactions post-traumatiques, à la détresse psychologique et au style d'attachement.

Afin de mieux déterminer s'il s'agit de sous-groupes vulnérables, nous avons poursuivi nos analyses en reconstituant les groupes et en créant un groupe manifestant des «réactions saines» $(n=55$, dont 21 ES et $34 \mathrm{EA}$ ) et un groupe présentant des «réactions complexes» $(n=14$, dont 10 ES et 4 EA) à l'échelle du deuil (Grief Experience Questionnaire).

Symptomatologie, style d'attachement, nature du décès (suicide vs maladie)

et ajustement au deuil « réactions

saines » vs « réactions complexes»

(voir tableau 3)

Pour la plupart des variables évaluées entre les deux sous-groupes (réactions saines et complexes) chez les participants endeuillés par suicide et ceux endeuillés par maladie, les anova simples ne révèlent
La symptomatologie et l'ajustement

au deuil (« deuil sain » vs « deuil

complexe ») (voir tableau 4)

Par la suite, indépendamment de la nature du décès, nous avons reformé deux nouveaux groupes selon leur ajustement au deuil (deuil sain vs deuil complexe) toujours en s'appuyant sur l'échelle du deuil (Grief Experience Questionnaire). Ces données nous démontrent que les participants présentant des «réactions saines» liées à leur deuil manifestent significativement moins de réactions dépressives, posttraumatiques et de détresse psychologique que les participants présentant des «réactions complexes». Il est important aussi de noter que le nombre de mois s'étant écoulé entre le décès et l'entrevue n'est pas statistiquement significatif $(p>0,05)$ entre les participants démontrant des réactions «saines» (50 mois) et les participants démontrant des réactions "complexes» (60 mois) liées au deuil.

\section{Symptomatologie et caractéristiques \\ influençant le développement \\ de complications à la suite du deuil \\ (voir tableau 5)}

Les résultats de la régression logistique (69 participants) indiquent que parmi toutes les variables incluses dans le modèle, seules les réactions post-traumatiques et le style d'attachement prédisent significativement l'ajustement au deuil («réactions saines» vs «réactions complexes » - voir le tableau 4 pour les détails des statistiques). Ainsi, la présence de réactions traumatiques plus intenses et un style d'attachement insécure sont des prédicteurs de réactions complexes à la suite du deuil.

pas d'association significative entre les groupes. Les seules différences significatives retrouvées concernent les symptômes dépressifs $(p=0,009)$ et post-traumatiques $(p=0,006)$ observés entre les deux sousgroupes (réactions saines et complexes) dans le groupe des endeuillés par suicide. Cette absence de différence significative auprès des autres données pourrait s'expliquer par un manque de puissance statistique étant donné le nombre restreint de participants dans chacun des sous-groupes (p. ex. sous-groupe d'endeuillés par maladie ayant un deuil complexe: 4 participants). De plus, il existe une association significative entre le style d'attachement sécure et les réactions de deuil saines tant chez les personnes endeuillées par suicide $(\mathrm{p}=0,003)$ que par maladie $(\mathrm{p}=0,000)$.

\section{DISCUSSION}

Les résultats indiquent que certains participants endeuillés, à la suite d'un décès par suicide, manifestent des préoccupations importantes, voire des réactions subjectives particulièrement au niveau des thématiques suivantes identifiées par les sous-échelles du questionnaire de deuil: la recherche d'explications, le sentiment de responsabilité, le sentiment de rejet et les réactions uniques. Cette donnée indique que ce deuil est qualitativement différent et s'avère particulier. Notre étude confirme les résultats de plusieurs études comparatives et/ou qualitatives portant sur les personnes endeuillées par suicide (Harwood et al., 2002; Callahan, 2000; Bailley et al.,1999; Clark et al.,1995; Séguin et al., 1995; Cleiren, et al.,1994; Valente et al.,1993; McIntosh et al., 1992; Van Dongen, 1991; Barrett et al.,1990; Ness et al., 1990; Van der Wal, 1989; 
TABLEAU 5

SYMPTOMATOLOGIE ET CARACTÉRISTIQUES ACCOMPAGNANT LE DÉVELOPPEMENT DE COMPLICATIONS À LA SUITE D'UN DEUIL

\begin{tabular}{|c|c|c|c|}
\hline & OR & $\mathrm{CI}_{95 \%}$ & $\mathrm{p}$ \\
\hline Réactions traumatiques & 1,20 & $1,06-1,36$ & .003 \\
\hline \multirow[t]{2}{*}{$\begin{array}{l}\text { Attachement } \\
\text { sécure/insécure }\end{array}$} & 11,24 & $2,05-61,67$ & .005 \\
\hline & \multicolumn{3}{|c|}{$\begin{array}{l}\text { Modèle: } \mathrm{x}^{2}(3)=25,20 \mathrm{p}=0,000 \\
\mathrm{R}^{2} \text { cox et snell }=0,310 ; \mathrm{R}^{2} \text { negelkerke }=0,485 \\
\text { Classification exacte du deuil compliqué } 50 \%\end{array}$} \\
\hline
\end{tabular}

* Seuls les résultats significatifs à $\mathrm{p}<0,05$ sont présentés.

Dunn et al.,1987-1988; Dunn et al., 1987 ; McIntosh, 1987; Sheskin et al.,1976).

La nature singulière du deuil par suicide de même que l'utilisation d'un questionnaire conçu pour les endeuillés par suicide et mesurant les réactions subjectives et non pas la symptomatologie des endeuillés peuvent expliquer des préoccupations qualitativement différentes chez les personnes endeuillées. Le suicide est souvent perçu comme une mort prématurée et injuste, puisqu'elle dévie du cours naturel des événements, d'où la difficulté «d'en trouver un sens». Par conséquent, la «recherche d'explications » prend forme par des ruminations mentales et un besoin compulsif de comprendre un tel geste. Cette difficulté se conjugue aussi avec l'impression que cette mort violente et soudaine aurait pu être évitée, d'autant plus qu'on en voit souvent des signes avant-coureurs. Les personnes endeuillées par suicide se remémorent l'événement rétrospectivement, ce qui peut les laisser avec une sensation d'avoir une participation active dans ce drame et provoquer un "sentiment de responsabilité ». Le «sentiment de rejet» est un autre aspect important observé dans nos résultats. Les personnes endeuillées par suicide peuvent être amenées à éprouver un sentiment de rejet, car le geste suicidaire étant intentionnel, il peut être perçu par elles comme un abandon délibéré. Les personnes endeuillées par suicide interprètent souvent le suicide comme une indication de leur importance aux yeux de la personne décédée (Séguin et al ., 1999). Enfin, la sous-échelle des «réactions uniques » évalue certains éléments plus spécifiquement liés au deuil par suicide (déni de la forme de décès, les motivations du défunt à poser cet acte, sentiment de vengeance envers l'endeuillé...). À la lumière des résultats, nous remarquons aussi que la sous-échelle «stigmatisation» ne se distingue pas significativement des deux groupes. Il s'agit pourtant d'une des dimensions les plus souvent répertoriées dans les études portant sur les endeuillés par suicide (Hanus, 2004). Il est toutefois possible qu'en raison du passage du temps (environ cinq ans) et de leur acceptation à participer à notre étude, ces endeuillés puissent être moins enclins à éprouver moins ce sentiment. Il se peut aussi que le suicide soit perçu moins négativement par la société québécoise, laissant par conséquent les personnes endeuillées par suicide moins craintives du jugement des autres et se sentant ainsi moins stigmatisées.

Malgré cette première observation quant à la présence de réactions qualitativement différentes chez les personnes endeuillées à la suite d'un suicide, nous n'observons pas de différences quant à la présence de symptomatologie plus importante entre les deux groupes. Ainsi la différence serait surtout au niveau des réactions subjectives liées au deuil, mais aucune différence n'est observée au niveau de la détresse psychologique, de la présence d'affects dépressifs, des réactions post-traumatiques ou des styles d'attachements.

Cependant, nos résultats indiquent que chez les participants endeuillés à la suite d'un suicide autant que chez ceux endeuillés par maladie, il y a présence de sous-groupes d'endeuillés manifestant des «réactions complexes». Tout comme avait pu l'observer Bonnano et al. (2004) auprès de divers groupes d'endeuillés, il existe des sous-groupes manifestant une détresse chronique, en dépit du passage du temps. C'est donc dire que ce ne sont pas tous les endeuillés par suicide qui seront amenés à vivre un deuil complexe. L'issue de l'ajustement à la perte n'est pas uniquement associée à la nature du décès, mais pourrait davantage être influencée par des variables préexistantes au décès. Ainsi plusieurs variables pourraient avoir un impact sur le deuil dont des événements s'inscrivant dans l'histoire personnelle et relationnelle de la personne endeuillée, tout comme l'ont aussi noté plusieurs études antérieures (Séguin et al.,1995a,1995b; Cleiren et al., 1994; Barrett et Scott,1990).
D'ailleurs, dans cette étude, le style d'attachement est l'une des variables les plus importantes pouvant influencer un ajustement plus complexe à la perte, indépendamment de la nature du décès. Les données de la régression logistique viennent mettre en lumière le fait qu'indépendamment de la nature du décès auquel sont confrontés les participants endeuillés, un style d'attachement insécure chez la personne endeuillée et la présence de réactions post-traumatiques importantes influencent grandement la présence de réactions complexes liées au deuil, évoquant la possibilité d'un ajustement au deuil plus difficile et plus long. Ces deux variables rejoignent les dimensions conceptuelles jusqu'à maintenant proposées par les équipes de recherche de Mardi J. Horowitz et de Selby Jacobs et Holly Prigerson, pour clarifier les critères du deuil compliqué (éléments de détresse, de séparation et de traumatisme) (Stroebe et al., 2000; Kim et al.,1991). En effet, le style d'attachement semble jouer un rôle considérable dans le développement d'un deuil pouvant s'avérer plus complexe à la suite du décès d'un être cher. Ce résultat n'est pas surprenant, étant donné que le processus de deuil est un travail de détachement qui repose sur un attachement préalable. Il semble donc qu'un style d'attachement insécure fasse souvent partie des vulnérabilités préexistantes à ce deuil et soit susceptible de raviver une anxiété de séparation chez l'endeuillé. La présence d'un style d'attachement insécure (Vanderwerker et al., 2006; Stroebe et al., 2005a, 2005b; Mulkulincer et al., 1993) chez certaines personnes pourrait les rendre plus fragilisées à éprouver des difficultés à s'adapter à des expériences de pertes subséquentes et les rendre possiblement plus à risque de développer des complications à la suite de leur deuil (Silverman et al., 2001; Séguin et al., 1995a; Séguin et al., 1994).

Chez certains, la trajectoire développementale est parsemée d'un lot important d'expériences d'adversité, telles que des soins inadéquats, des pertes précoces, des abus, de la négligence (Silverman, Johnson et Prigerson, 2001) et la présence d'anxiété de séparation dès l'enfance (Vanderwerker, Jacobs, Parkes et Prigerson, 2006). Liée étroitement au spectre de ces expériences, la présence d'un style d'attachement insécure (Vanderwerker et al., 2006; Stroebe et al., 2005a, 2005b; Mulkulincer et al., 1993) et la présence d'antécédents de troubles de santé mentale (Brent et Melhem, 2008; Stenager et Qin, 2008; Tsuchiya et al., 2005; Jordan, 2001; Egeland et al., 1990) chez certaines personnes endeuillées pourraient les prédis- 
poser, en raison de ces facteurs de risque, à éprouver des difficultés à s'adapter à des expériences de pertes subséquentes et à développer des complications à la suite de leur deuil (Silverman et al., 2001; Séguin et al., 1995 ; Séguin et al., 1994).

Par ailleurs, le fait que les réactions traumatiques contribuent au développement de réactions complexes liées au deuil est intéressant. En effet, il a été suggéré que le décès d'un être cher pourrait s'inscrire comme une expérience qui n'est pas correctement régulée et qui, par conséquent, serait susceptible d'envahir une bonne partie de la vie psychique et d'être réactivée lors d'émotions intenses (Shore, 2008). Nous remarquons que les personnes constituant le groupe d'endeuillés ayant un deuil complexe ont rapporté plus de réactions traumatiques que les endeuillées vivant un deuil sain. Nous ne disposons pas de données concernant l'exposition ou non à la scène traumatique lors du décès chez les personnes endeuillées par suicide. Par conséquent, il est fort possible qu'un certain nombre d'entre elles aient été témoins de cette scène, les laissant hantées par des images intrusives et troublantes les rendant susceptibles d'éprouver des réactions de stress post-traumatique, ceci tant chez le groupe de personnes endeuillées à la suite d'un suicide que le groupe d'endeuillées à la suite d'une maladie.

Finalement, les résultats de notre étude suggèrent que les endeuillés vivant un deuil complexe seront plus à risque de vivre d'autres complications comme des réactions dépressives, post-traumatiques et de la détresse psychologique de façon plus importante que ceux vivant un deuil plus sain.

\section{LES LIMITES MÉTHODOLOGIQUES}

Cette étude exploratoire a des limites méthodologiques importantes, dont la petite taille de l'échantillon, ce qui limite la généralisation des résultats. Toutefois, le nombre de participants est semblable à celui d'autres études (Mitchell et al., 2004; Kaltman et al., 2003; Grad et al.,1999; Cleiren et al.,1996; Séguin et al., 1995a, 1995b; Silverman et al.,1994-1995; Barrett,1990; Range et al.,1990; Calhoun et al.,1984; Sanders, 1983). Le calcul de puissance révèle que le nombre de participants était suffisant pour détecter les tailles d'effets anticipés.

Le délai entre le décès et l'entretien avec les participants est une autre limite dont il faut tenir compte. Ce délai est variable (de deux à sept ans) entre les participants, mais il constitue cependant une période après laquelle le deuil est relativement résolu. Très peu d'études sur le deuil ont

étudié la présence de complications après sept ans (Lehman et al.,1987).

Nous n'avons pas évalué, chez les participants endeuillés de l'étude, la présence d'autres événements de vie importants,

SUR LE PLAN CLINIQUE, ÉTANT DONNÉ QUE LE DEUIL NE SE DÉPOSE PAS SUR UN TERRAIN VIERGE, IL SERAIT IMPORTANT DE POUVOIR EXPLORER ET DE BIEN ÉVALUER, CHEZ L'ENDEUILLÉ, CERTAINES VARIABLES ANTÉRIEURES AU DÉCÈS TELLES QUE LE STYLE D'ATTACHEMENT, LES ANTÉCÉDENTS DE TROUBLES DE SANTÉ MENTALE, LE STYLE D'ATTACHEMENT ET LA NATURE DE LA RELATION, LESQUELLES POURRAIENT PRÉDIRE DES DIFFICULTÉS

À S'AJUSTER AU DEUIL...

depuis le décès. En raison du délai passablement long entre le décès et l'entrevue, il est difficile de déterminer si certaines réactions sont consécutives au décès ou liées à d'autres événements qui auraient pu survenir depuis le décès ou même l'avoir précédé.

Malgré le fait que le deuil constitue en lui-même une expérience éprouvante, son évolution et son issue peuvent différer d'un individu à un autre. Il est important de se rappeler que la nature du décès ne semble pas être la variable pouvant, à elle seule, expliquer l'ajustement ultérieur au deuil. En nous appuyant sur les résultats de cette étude, nous constatons que l'évolution du processus de deuil est considérablement différente en fonction de certains sousgroupes de personnes endeuillées, et ceci, indépendamment de la nature du décès. Sur le plan clinique, étant donné que le deuil ne se dépose pas sur un terrain vierge, il serait important de pouvoir explorer et de bien évaluer, chez l'endeuillé, certaines variables antérieures au décès telles que le style d'attachement, les antécédents de troubles de santé mentale, le style d'attachement et la nature de la relation, lesquelles pourraient prédire des difficultés à s'ajuster au deuil...

Comme nous l'avons observé dans cette étude, un deuil complexe peut s'accompagner aussi de réactions dépressives, posttraumatiques et de détresse psychologique plus élevées. Dans des recherches ultérieures, il serait intéressant d'évaluer si ces réactions sont réellement liées au deuil ou si elles étaient présentes avant le décès. De plus, le terrain sur lequel se dépose le deuil peut aussi évoluer et exposer la personne endeuillées à de nombreuses difficultés survenant à la suite du décès, ces variables mériteraient également d'être mieux circonscrites. Ainsi, les recherches ultérieures pourraient considérer et évaluer la capacité de mentalisation et les 
BRENT, D. A. et MELHEM, N. (2008). "Familial transmission of suicidal behavior ». Psychiatric Clinics of North America, vol. 31, n 2, p. 157-177.

BRUNET, A., ST-HILAIRE, A., JEHEL, J. et KING., S. ((2003)) «Validation of a French version of the impact of event scale-revised». Canadian Journal of Psychiatry, vol. $48, \mathrm{n}^{\circ} 1$, p. 56-61.

CALHOUN, L. G., SELBY, J. W. et ABERNATHY., C. B. (1984). "Suicidal death: Social reactions to bereaved survivors ». The Journal of Psychology, vol. 116, $2^{\mathrm{e}}$ partie, p. 255-261.

CALHOUN, L. G., SELBY, J. W. et SELBY., L. E. (1982). "The psychological aftermath of suicide: An analysis of current evidence ». Clinical Psychology Review, vol. 2, $\mathrm{n}^{\circ} 3$, p. 409-420.

CALLAHAN, J. (2000). "Predictors and correlates of bereavement in suicide support group participants». Suicide Life-Threatening Behavior, vol. 30, $\mathrm{n}^{\circ}$ 2, p. 104-124.

CLARK, S. E. et GOLDNEY., R. D. (1995). "Grief reactions and recovery in a support group for people bereaved by suicide». Crisis: The Journal of Crisis Intervention and Suicide Prevention, vol. 16, $\mathrm{n}^{\circ}$ 1, p. 27-33.

CLEIREN, M. P. (1993). "Bereavement and adaptation: A comparative study of the aftermath of death ». Series in death education, aging, and health care, Washington, DC: Hemisphere Publishing Corp.

CLEIREN, M. P., GRAD, O., ZAVASNIK, A. et DIEKSTRA, R. F. (1996). "Psychosocial impact of bereavement after suicide and fatal traffic accident: A comparative two-country study». Acta Psychiatrica Scandinavica, vol. $94, n^{0} 1$, p. 37-44.

CLEIREN, M. P. H. D. et DIEKSTRA, R. F. W. (1995). «After the loss: bereavement after suicide and other types of death». In The Impact of Suicide. Éd. de B. Mishar, p. 7-39. New York: Springer.

CLEIREN, M. P. H. D., DIEKSTRA, R. F. W., KERKHOF, A .J. F. M. et VAN DER WAL. J. (1994). "Mode of death and kinship in bereavement: Focusing on 'who' rather than 'how.' ». Crisis: The Journal of Crisis Intervention and Suicide Prevention, vol. $15, \mathrm{n}^{\circ} 1$, p. 22-36.

CUILLERIER, L.-M. (2004). «Le trouble de stress post-traumatique: L'échelle de l'effet des événements révisée ». <http://instrumentspsychometriques.mcgill.ca/>

DE GROOT, M. H., DE KEIJSER, J. et NEELEMAN, J. (2006). «Grief shortly after suicide and natural death: A comparative study among spouses and first-degree relatives ». Suicide and Life Threatening Behavior, vol. 36, p. 418-431.

DE MONTIGNY, J. (2002). "Quand le deuil se complique». Psychologie Québec, novembre, p. 19-22.

DEMI, A. S. (1984). «Social adjustment of widows after a sudden death: Suicide and non-suicide survivors compared». Death Education, vol. 8 (Suppl.), p. 91-111.
DEMI, A. S. et HOWELL, C. (1991). «Hiding and healing: Resolving the suicide of a parent or sibling». Archives of Psychiatric Nursing, vol. $5, \mathrm{n}^{\circ} 6$, p. 350-356.

DUNN, G. R. et MORRISH-VIDNERS, D. (1987)-(1988). "The psychological and social experience of suicide survivors". Omega: Journal of Death and Dying, vol. 18, $\mathrm{n}^{\circ}$ 3, p. 175-215.

DYREGROV, K., NORDANGER, D., ET DYREGROV, A. (2003). "Predictors of psychosocial distress after suicide, SIDS and accidents ». Death Studies, vol. 27, $\mathrm{n}^{\circ} 2$, p. 143-165.

EGELAND, J. A., SUSSEX, J. N., ENDICOTT, J. et HOSTETTER, A.M. (1990). "The impact of diagnoses on genetic linkage study for bipolar affective disorders among the Amish ». Psychiatric Genetics, vol. 1, p. 5-18.

ELLENBOGEN, $S$. et GRATTON, F. (2001). «Do they suffer more? Reflections on research comparing suicide survivors to other survivors». Suicide et Life-Threatening Behavior, vol. 31, $\mathrm{n}^{\circ}$ 1, p. 83-90.

ENRIGHT, B. P. et MARWIT, S .J. (2002). «Diagnosing complicated grief: A closer look». Journal of Clinical Psychology, vol. $58, \mathrm{n}^{\circ} 7$, p. 747-757.

FARBEROW, N. L., GALLAGHER, D. E., GILEWSKI, M. J. et THOMPSON, L.W. (1987). «An examination of the early impact of bereavement on psychological distress in survivors of suicide ». Gerontologist, vol. 27, $\mathrm{n}^{\circ} 5$, p. 592-598.

FARBEROW, N. L., GALLAGHER-THOMPSON, D. E., GILEWSKI, M. J. et THOMPSON, L. W. (1992). "Changes in grief and mental health of bereaved spouses of older suicides ». Journal of Gerontology, vol. 47, no 6, p. 357-366.

FIELD, N.P. et E.C. SUNDIN (2001). "Attachment style in adjustment to conjugal bereavement», Journal of Social and Personal Relationships, vol. 18, p. 347-361.

FRALEY, R. C., ET BONANNO, G. A. (2004). "Attachment and loss: A test of three competing models on the association between attachment-related avoidance and adaptation to bereavement». Personality and Social Psychology Bulletin, vol. 30, $\mathrm{n}^{\circ}$ 7, p. 878-890.

GAUTHIER, J., MORIN, C., THÉRIAULT, F. et LAWSON, J. S. (1982). "Adaptation française d'une mesure d'auto-évaluation de l'intensité de la dépression ». Revue québécoise de psychologie, vol. 3, p. 13-27.

GRAD, O.T. et ZAVASNIK, A. (1999). «Phenomenology of bereavement process after suicide, traffic accident and terminal illness (in spouses) ». Archives of Suicide Research, vol. $5, \mathrm{n}^{\circ} 2$, p. 157-172.

GRIFFIN, D. W. et BARTHOLOMEW., K. (1994). "Models of the self and other: Fundamental dimensions underlying measures of adult attachment». Journal of Personality and Social Psychology, vol. 67, $\mathrm{n}^{\circ} 3$, p. 430-445.
HANUS, M. (2004). « Le deuil après suicide est-il particulier? ». In Le deuil après suicide (chap.15), Paris: Maloine, p. 263-276.

HARWOOD, D., HAWTON, K., HOPE, T. et JACOBY, R. (2002). "The grief experiences and needs of bereaved relatives and friends of older people dying through suicide: A descriptive and case-control study ". Journal of Affective Disorders, vol. 72, $\mathrm{n}^{\circ} 2$, p. 185-194.

HAZAN, C., ET SHAVER., P. (1987). "Romantic love conceptualized as an attachment process». Journal of Personality and Social Psychology, vol. 52, $\mathrm{n}^{\circ}$ 3, p. 511-524.

ILFELD, F.W. (1976). «Further validation of a psychiatric symptom index in a normal population ", Psychological Reports, vol. 39, n 3, p. 1215-1228.

INSTITUT NATIONAL DE SANTÉ PUBLIQUE DU QUÉBEC. ((2005)). La mortalité par suicide au Québec: tendances et données récentes. Québec: Publications du Québec.

JORDAN, J. R. (2001). «Is suicide bereavement different? A reassessment of the literature ». Suicide et Life-Threatening Behavior, vol. 31, n 1, p. 91-102.

KALTMAN, S., ET BONANNO, G. A. (2003). "Trauma and bereavement: Examining the impact of sudden and violent deaths». Journal of Anxiety Disorders, vol. 17, no 2, p. 131-147.

KIM, K., ET JACOBS, S. (1991). «Pathologic grief and its relationship to other psychiatric disorders ». Journal of Affective Disorders, vol. $21, n^{\circ} 4$, p. $257-263$.

KITSON, G. (2000). «Adjustment to violent and natural deaths in later and earlier life for black and white widows». Journal of Gerontology: Series B: Psychological Sciences and Social Sciences, vol. 55B, $\mathrm{n}^{\circ} 6$, p. 341-351.

KNIEPER, A. J. (1999). "The suicide survivor's grief and recovery». Suicide \& Life-Threatening Behavior, vol. 29, $\mathrm{n}^{\circ} 4$, p. 353-364.

LEHMAN, D. R., WORTMAN, C. B., ET WILLIAMS, A. F. (1987). "Long-term effects of losing a spouse or child in a motor vehicule crash ». Journal of Personality and Social Psychology, vol. 52, p. 218-231.

MCINTOSH, J. (1987). "Survivors family relationships: literature review». In Suicide and its aftermath. Understanding and counselling the survivors. Éd. d'E. J. Dunne, et al., p. 73-84.New York: W.W. Norton,

MCINTOSH J. L. et KELLY, L. D. (1988). "Survivors' reactions: Suicide vs other causes». Communication présentée au $21^{\text {e }}$ Congrès annuel de l'American Association of Suicidology, Washington DC. In Impact du suicide sur l'entourage et aspects neurobiologiques du deuil document, C. Sève (2009), PDF, consulté le 23 avril 2011.

MCINTOSH, J. et KELLY, L. D. (1992). "Survivors' reactions: Suicide vs. other causes ». Crisis, vol. 13, n 2, p. 82-93. 
MCNIEL, D. E., HATCHER, C. et REUBIN, R. (1988). "Family survivors of suicide and accidental death: consequences for widows ». Suicide et Life-Threatening Behavior, vol. $18, \mathrm{n}^{\circ} 2$, p. 137-148.

MITCHELL, A. M., KIM, Y. J., PRIGERSON, H. G. et MORTIMER-STEPHENS, M. (2004). "Complicated grief in survivors of suicide». Crisis, vol. $25, \mathrm{n}^{\circ}$ 1, p. 12-18.

MULKULINCER, M., FLORIAN, V. etWELLER, A. (1993). "Attachment styles, coping strategies, and posttraumatic psychological distress: The impact of the Gulf war in Israel ». Journal of Personality and Social Psychology, vol. 64, $\mathrm{n}^{\circ}$ 5, p. 817-826.

MURPHY, S. A., JOHNSON, L. C., CHUNG, I. J. et BEATON, R. D. (2003). "The prevalence of PTSD following the violent death of a child and predictors of change 5 years later». Journal of Traumatic Stress, vol. 16, $\mathrm{n}^{\mathrm{o}} 1$, p. 17-25.

NESS, D. E., ET PFEFFER, C.R. (1990). «Sequelae of bereavement resulting from suicide». American Journal of Psychiatry, vol. $147, \mathrm{n}^{\circ} 3$, p. $279-285$.

OSTERWEIS, M. (1984). «Réactions to particular types of bereavement». In Bereavement, reactions, consequences and care. Éd. de M. Osterweis et al., p. 83-92. Washington DC: Academy Press.

PRÉVILLE, M., BOYER, R., POTVIN, L., PERRAULT, C. et LEGARÉ, G. (1992). «La détresse psychologique: détermination de la fiabilité et de la validité de la mesure utilisée dans l'enquête Santé Québec ». Québec, Rapport de recherche soumis à Santé Québec.

PRIGERSON, H. G., FRANK, E, KASL, S. V., REYNOLDS, C. F., ANDERSON, B., ZUBENKO, G. S., ET AL. (1995). "Complicated grief and bereavement-related depression as distinct disorders: Preliminary empirical validation in elderly bereaved spouses». American Journal of Psychiatry, vol. 152, p. 22-30.

PRIGERSON, H. G., SHEAR, M. K., JACOBS, S. C., REYNOLDS, C. F., MACIEJEWSKI, P. K., ET AL. (1999). «Consensus criteria for traumatic grief: a preliminary empirical test ». British Journal of Psychiatry, vol. 174, p. 67-73.

RANGE, L. M., ET NISS., N.M. (1990). «Long-term bereavement from suicide, homicide, accidents, and natural deaths». Death Studies, vol. 14, no 5, p. 423-433.

REED, M. D. (1998). «Predicting grief symptomatology among the suddenly bereaved ». Suicide and Life-Threatening Behavior, vol. 28, n 3, p. 285-301.

REED, M. D. et GREENWALD., J. Y. (1991). "Survivor-victim status, attachment, and sudden death bereavement». Suicide and Life-Threatening Behavior, vol. 21, $\mathrm{n}^{\circ} 4$, p. 385-401.

RUDESTAM, K. E. (1977). «Physical and psychological responses to suicide in the family ». Journal of Consulting and Clinical Psychology, vol. 45, n 2, p. 162-170.

SANDERS, C. M. (1983). «Effects of sudden versus chronic illness death on bereavement outcome». Omega: Journal of Death and Dying, vol. 13, n 3, p. 227-241.

SCHARFE, E. et BARTHOLOMEW., K. (1994). "Reliability and stability of adult attachment patterns». Personal Relationships, vol. 1, n ${ }^{\circ} 1$, p. 23-43.

SÉGUIN, M. et CASTELLI-DRANSART, D. A. (2006). «Le deuil suite à un suicide: symptomatologie et choix d'intervention ». Traité de Psychiatrie et de pédo-psychiatrie de l'Encyclopédie Médico-Chirurgicale (EMC), Paris, Elsevier-Masson.

SÉGUIN, M. et FRÉCHETTE, L. (1999). Le deuil: une souffrance à comprendre pour mieux intervenir, Montréal: Logiques.

SÉGUIN M., KIELY, M. et LESAGE., A. (1994). "L'après-suicide: une expérience unique de deuil?". Santé Mentale au Québec, vol. 19, p. 63-82.

SÉGUIN, M., LESAGE, A. et KIELY, M. (1995a). "History of early loss among a group of suicide survivors ». Crisis, vol. 16, $\mathrm{n}^{\circ} 3$, p. 121-125.

SÉGUIN, M., LESAGE, A. et M.C. KIELY. (1995b). "Parental bereavement after suicide and accident: A comparative study». Suicide and Life-Threatening Behavior, vol. 25, $n^{\circ} 4$, p. 489-492.

SHEPHERD, D. et BARRACLOUGH, B. M. (1974). "The aftermath of suicide». British Medical Journal, vol. 2, $\mathrm{n}^{\circ}$ (5919), p. 600603.

SHNEIDMAN, E. S. (1969). "Prologue». In On the Nature of Suicide, sous la direction d'E.S. Shneidman, San Francisco: Jossey-Bass.

SHORE, A. (2008). La régulation affective et la réparation du soi. Montréal: Éditions du CIG.

SILVERMAN, E., RANGE, L. M. et OVERHOLSER, J. C. (1994)-(1995). «Bereavement from suicide as compared to other forms of bereavement». Omega: Journal of Death and Dying, vol. 30, $\mathrm{n}^{\circ} 1$, p. 41-45.

SILVERMAN, G. K., JOHNSON, J. G. et PRIGERSON, H. G. (2001). "Preliminary explorations of the effects of prior trauma and loss on risk for psychiatric disorders in recently widowed people ». Israel Journal of Psychiatry, vol. 38, p. 202-215.

SIMPSON, J. A., RHOLES, W.S. et NELLIGAN, J.S. (1992). "Support seeking and support giving within couples in an anxiety-provoking situation: The role of attachment styles». Journal of Personality and Social Psychology, vol. 62, n 3, p. 424-446.

STENAGER, K. et QIN, P. (2008). «Individual and parental psychiatric history and risk for suicide among adolescents and young adults in Denmark: A population-based study». Social Psychiatry and Psychiatric Epidemiology, vol. 43, n ${ }^{\circ}$ 11, p. 920-926.

STROEBE, M. S., SCHUT, H., ET STROEBE, W. (2005a). "Attachment in coping with bereavement: A theoretical integration». Review of General Psychology, vol. 9, p. $48-60$.

STROEBE, M. S., SCHUT, H. et STROEBE, W. (2005b). «Who benefit from disclosure?
Exploration of attachment style differences in the effect of expressing emotions ». Clinical Psychology Review, vol. 26, p. 66-85.

STROEBE, M., VAN SON, M., STROEBE, W., KLEBER, R., SCHUT, H. et VAN DEN BOUT., J. (2000). «On the Classification and diagnosis of pathological grief ». Clinical Psychology Review, vol. 20, $\mathrm{n}^{\circ}$ 1, p. 57-75.

SVEEN, C. A. et WALBY, F.A. (2008). "Suicide survivors' mental health and grief reactions: A systematic review of controlled studies». Suicide Life-Threatening Behavior, vol. 38, n 1 , p. 13-29.

TSUCHIYA, K. J., AGERBO, E. et MORTENSEN, P.B. (2005). «Parental death and bipolar disorder: A robust association was found in early maternal suicide». Journal of Affective Disorders, vol. 86, $\mathrm{n}^{\circ} 2-3$, p. 151-159.

VALENTE, S. M. et SAUNDERS, J.M. (1993). "Adolescent grief after suicide». Crisis, vol. 14, no 1, p. 22-46.

VALLERAND, R. J. (1989). "Vers une méthodologie de validation transculturelle de questionnaires psychologiques: Implication pour la recherche en langue française». Psychologie canadienne, vol. 30, $\mathrm{n}^{\circ} 4$, p. 662-680.

VAN DER WAL, J. (1989)-(1990). "The aftermath of suicide: A review of empirical evidence». Omega: Journal of Death and Dying, vol. 20, $\mathrm{n}^{\circ} 2$, p. 149-171.

VANDERWERKER, L. C., JACOBS, S.C., PARKES, C.M. et PRIGERSON, H.G. (2006). "An exploration of associations between separation anxiety in childhood and complicated grief in later life». The Journal of Nervous and Mental Disease, vol. 194, n 2, p. 121-123.

VAN DONGEN, C. J. (1991). «Experiences of family members after a suicide». The Journal of Family Practice, vol. 33, $\mathrm{n}^{\circ} 4$, p. 375-380.

VAN DOORN, C., KASL, S.V., BEERY, L.C., JACOBS, S.C. et PRIGERSON, H.G. (1998). "The influence of marital quality and attachment styles on traumatic grief and depressive symptoms ». .Journal of Nervous and Mental Disease, vol. 186, n ${ }^{\circ}$ 9, p. 566573.

WAYMENT, H. A. et VIERTHALER, J. (2002). «Attachment style and bereavement reaction ». Journal of Loss and Trauma, vol. 7, p. 129-149. 